\title{
Exploiting asymmetric signals from choices through default selection
}

\author{
Lim M. Leong ${ }^{1}$ (D) Yidan Yin ${ }^{2} \cdot$ Craig R. M. McKenzie ${ }^{1,2}$
}

Published online: 2 January 2020

(C) The Psychonomic Society, Inc. 2019

\begin{abstract}
Setting defaults is an effective nudge, but few studies have examined situations where individuals can select their own default settings. Past research suggests that even when the final outcome is identical, observers perceive stronger signals from choices that switch from, rather than stay with, the default. In five experiments using hypothetical scenarios and an incentivized economic game, we test whether decision-makers driven by image concerns could strategically exploit that asymmetric signal. We found that in the presence of observers, participants were more likely to self-select into defaults that require them to switch to enhance a positive signal and into defaults that require them to stay to attenuate a negative signal. Our results support the framework of choice architecture as an implicit social interaction, and have potential implications for behavioral interventions in real-world settings.
\end{abstract}

Keywords Choice architecture $\cdot$ Nudges $\cdot$ Default options $\cdot$ Behavioral signaling

Setting default options is a potent and popular behavioral intervention for choice architects (Benartzi et al., 2017; Thaler $\&$ Sunstein, 2008). Designating the option you want others to choose as the default-namely, the one that is implemented when decision-makers (DMs) fail to make an active choicecan increase the chances that it is chosen. For example, organ donation consent rates are higher in countries where residents are presumed to be donors by default than countries where they are not (Johnson \& Goldstein, 2003). Because defaults do not alter the menu of options, they allow choice architects to nudge DMs toward desired outcomes while preserving their autonomy to choose.

The existing research on default effects has focused almost exclusively on how choices are affected by different randomly assigned defaults. What if DMs can instead choose which default setting to interact with? For instance, imagine that

Preregistration documents, materials, and data can be found at the Open Science Framework page for this article (https://osf.io/d94xn/).

Electronic supplementary material The online version of this article (https://doi.org/10.3758/s13423-019-01699-2) contains supplementary material, which is available to authorized users.

Lim M. Leong

lmleong@ucsd.edu

1 Department of Psychology, UC San Diego, La Jolla, CA, USA

2 Rady School of Management, UC San Diego, La Jolla, CA, USA different restaurants have set different side dishes as the default, and you are choosing which restaurant to take your friend out for dinner. You want to impress your friend by showing that you eat healthily so you would obviously order salad over fries. But would you prefer to order that salad at a restaurant where salad is the default or at one where fries is the default? As we explain below, we suspect that many would prefer the latter restaurant, where you would actively order the salad rather than passively receive it. In the experimental paradigm of the present research, we allow DMs to choose between different default settings to obtain the same options.

A key factor that may drive DMs to self-select into different default settings is social image concerns. Although defaults do not change the availability of options, they can affect the social meaning attached to those options (Krijnen, Tannenbaum, \& Fox, 2017). That is, the same option can send different signals depending on whether it was obtained by staying with, or switching from, the default. To illustrate, participants in one experiment were asked to rate how altruistic it would be to donate organs under different default policies (Davidai, Gilovich, \& Ross, 2012). Participants who read that the country had a "not an organ donor" default judged organ donation as comparable to bequeathing half of one's wealth to charity, but those who read that the country had an "organ donor" default judged organ donation to be akin to letting others go ahead in line. Similarly, participants playing the role of a surviving family member inferred that a decedent on the organ donor register has a stronger underlying preference to donate when the choice was made under an opt-in system than opt- 
out (Lin, Osman, Harris, \& Read, 2018). Organ donation was perceived as much more meaningful and impactful, and as a stronger indicator of one's true preference, when it required switching from the default.

Given that observers perceive asymmetric signals from choices under different default regimes, we examine whether DMs can strategically exploit this perception. Our main hypothesis is that DMs motivated to send a stronger positive signal will self-select into the default regime that requires them to switch from the default option. Recent findings provide conflicting evidence on whether DMs are capable of such strategic behavior. Zlatev, Daniels, Kim, and Neale (2017) claim that in attempts at social influence, DMs often exhibit "default neglect," or the inability to set optimal defaults. Recent conceptual replications, however, suggest that this poor performance is limited to the original materials and that DMs are instead very good at setting defaults (Jung, Sun, \& Nelson, 2018; McKenzie, Leong, \& Sher, 2019). Our research question requires even more complicated reasoning about defaults, and if participants indeed do not understand how defaults influence others' choices, then they should similarly fail to self-select into optimal default regimes.

\section{Experiment 1}

We first attempted to conceptually replicate the finding that observers perceive a stronger signal from switching than staying with the default in a healthy eating scenario. To this end, we placed participants in the role of observers and asked them to draw inferences about two friends: One who ordered salad at a restaurant where salad is the default, and the other who ordered salad where fries is the default. We predicted that participants would infer that the friend who ordered salad at the fries default restaurant cared more about healthy eating.

\section{Method}

We used a rule of thumb and decided in advance to set the target sample size to be 100 participants per between-subjects condition. A total of 225 undergraduates in the UCSD Psychology Department ( $M_{\mathrm{age}}=19.56$ years; $77 \%$ female) participated for partial course credit. After participants completed one of our experiments, they were barred from signing up for all subsequent ones. Participants read two scenarios about going out to dinner with a friend, and in each scenario, the friend ended up choosing a salad. In one scenario, the restaurant had salad as the default side dish, but customers could switch to get fries. In the other scenario, the restaurant had fries as the default side dish, but customers could switch to get salad. Thus, the friend in one scenario chose salad by staying with the default, and the friend in the other scenario chose salad by switching from the default. The order of presentation for the two scenarios was manipulated between subjects. For each scenario, participants rated how much they thought that their friend "truly cares about eating healthy" on a scale from 1 (not at all) to 9 (very much). The participants' rating to this question for the first scenario was displayed to them when they made their ratings for the second scenario. For all experiments, we have reported all conditions, data exclusions, and our main independent and dependent measures. ${ }^{1}$

\section{Results}

Figure 1 shows the mean ratings of healthy eating for the friend who chose salad under different defaults and presentation orders. A mixed ANOVA was performed on the ratings, with order as a between-subjects factor and default as a within-subjects factor. This analysis revealed a main effect of default, $F(1,223)=159.89, p<.001, \eta_{\mathrm{g}}{ }^{2}=.182$, as well as a significant interaction, $F(1,223)=26.36, p<.001, \eta_{\mathrm{g}}{ }^{2}=$ .035. Participants rated the friend who ordered salad at the fries default restaurant, $M=6.92, S D=1.70$, as caring more about healthy eating than the friend who ordered salad at the salad default restaurant, $M=5.31, S D=1.85$. The magnitude of this difference, however, depended on the presentation order. Additional paired-sample $t$ tests showed that the difference in ratings was larger when participants read the salad default scenario first, $M \mathrm{~s}=7.58$ vs. $5.31, M_{\text {diff }}=2.27, S D_{\text {diff }}$ $=1.89, t(112)=12.74, p<.001, d=1.20,95 \%$ CI $[1.91,2.62]$, compared with when they read the fries default scenario first, $M \mathrm{~s}=6.27$ vs. $5.31, M_{\text {diff }}=0.96, S D_{\text {diff }}=1.94, t(111)=5.22, p$ $<.001, d=0.49,95 \%$ CI $[0.59,1.32]$. To examine whether this pattern holds in a between-subjects design, we compared the participants' ratings of the first scenario they were presented with. An independent $t$ test showed a higher mean rating of healthy eating for the friend who ordered salad at the fries default restaurant compared with the one who did so at the salad default restaurant, $M_{\text {fries }}=6.27, S D_{\text {fries }}=1.54, M_{\text {salad }}=$ $5.31, S D_{\text {salad }}=1.85, t(216.3)=4.23, p<.001, d=0.56,95 \%$ CI $[0.51,1.41]$. Together, these results confirm our prediction that ordering salad by switching from the fries default, rather than staying with the salad default, was perceived as a stronger signal of being a healthy eater.

\section{Experiments 2a-b}

The previous experiment found that observers perceived switching from the fries default to order salad as a stronger signal of being a healthy eater. In the next two experiments, we placed participants in the opposite role: DMs could choose

\footnotetext{
${ }^{1}$ See Supplementary Material for our additional dependent variable and its analysis.
} 


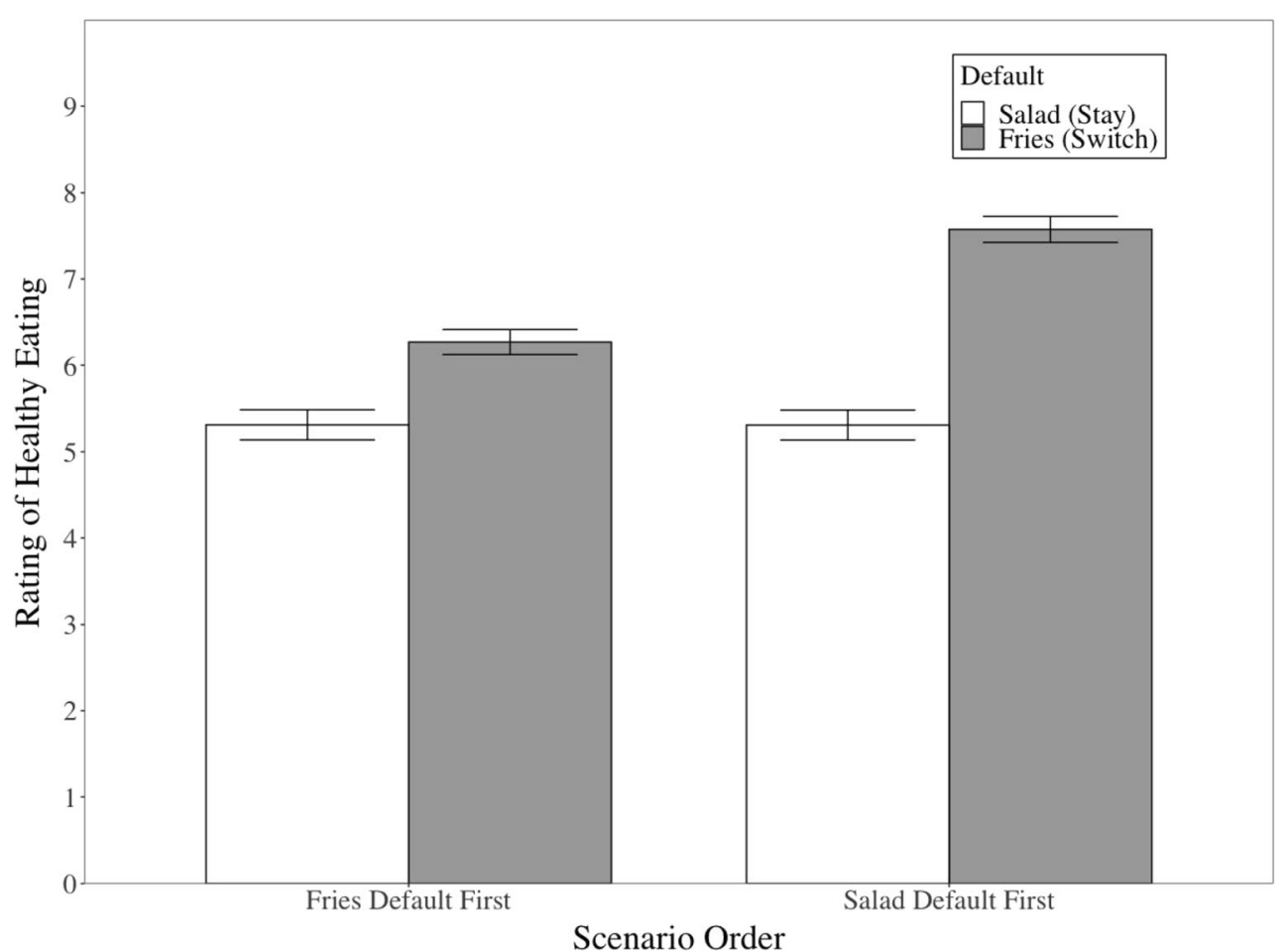

Fig. 1 Mean ratings of healthy eating as a function of side dish default and scenario presentation order in Experiment 1. Error bars represent standard error of the mean

which restaurant to take their friend out for dinner. Experiment 2a provided an initial test, while Experiment $2 \mathrm{~b}$ replicated and extended it by manipulating the visibility of choice to test whether choices are driven by behavioral signaling. We predicted that DMs would self-select into the fries default restaurant to order salad only in the presence of an observer.

\section{Method}

We decided in advance to set our target sample size to be 100 participants per between-subjects condition. A total of 136 undergraduates participated in Experiment 2a $\left(M_{\text {age }}=19.88\right.$; $70 \%$ female), and a total of 202 undergraduates participated in Experiment $2 \mathrm{~b}\left(M_{\mathrm{age}}=20.00 ; 63 \%\right.$ female, 1 reported "Other"). All participants received partial credit in psychology courses.

Experiment 2a had only one condition. Participants imagined going out to dinner with a friend and could choose one of two restaurants to order a salad. The restaurants were said to be comparable in every way and offered the same choice set, except fries come on the side by default at one and salad comes on the side by default at the other. Furthermore, the friend has never heard of these restaurants, and would only know about the one that participants decide on. Finally, participants were told that they want to impress their friend by showing that they eat healthily and were asked to indicate which restaurant they would choose to take their friend.
In Experiment 2b, participants were randomly assigned to either the "public" or "private" condition. The "public" condition is a direct replication of Experiment 2a, and participants again imagined that they were going out to dinner with a friend whom they want to impress. In the "private" condition, participants chose between the same two restaurants, but imagined that they were going out to dinner alone and that they were conscious about their health.

\section{Results}

Figure 2 shows the percentage of participants who chose the restaurant with the fries default for Experiments $2 \mathrm{a}$ and $2 \mathrm{~b}$. When dining with a friend, $71.3 \%$ (97/136) of participants in Experiment $2 \mathrm{a}$ chose the fries default restaurant, which was significantly higher than chance, $\chi^{2}(1, N=136)=23.89, p<$ $.001,95 \%$ CI $[62.8 \%, 78.6 \%]$. In Experiment 2b, 76\% (77/ 101) of participants in the "public" condition similarly chose the fries default restaurant, but only $12 \%(12 / 101)$ of participants in the "private" condition chose the fries default restaurant, $\chi^{2}(1, N=202)=82.27, p<.001, \varphi=.64$. These results suggest that motivation to signal is a key factor that drives selection into different default regimes. When there was an opportunity to signal, participants strategically chose the default setting with the intention of switching away from the default. By doing so, their combination of default and choice matched the one that observers perceived as a stronger signal of being a healthy eater. When there was no opportunity to 


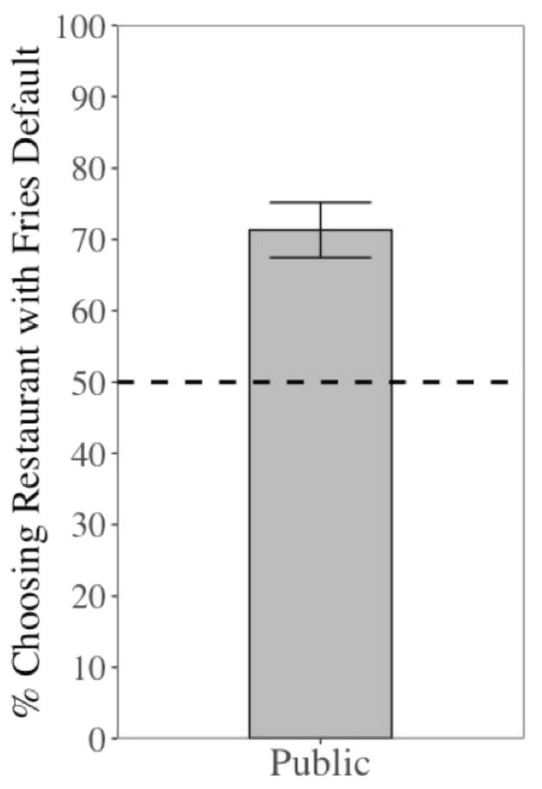

Experiment 2a

Fig. 2 The percentage of participants who chose the restaurant with the fries default to order a salad in Experiments $2 \mathrm{a}$ and $2 \mathrm{~b}$. Experiment $2 \mathrm{a}$ only has the public condition, while Experiment $2 b$ manipulated the

signal, however, participants simply chose the default setting that matched their choice.

\section{Experiment 3}

Thus far, we have tested our research question using only hypothetical scenarios. In our next experiment, we adapted the paradigm of Pleasant and Barclay (2018) and used incentivized economics games through which participants compete to be chosen by observers for a subsequent task. Specifically, participants played a modified Dictator Game and could strategically self-select into a fair split default or a selfish split default. As before, we manipulated the visibility of choice to test whether signaling drives their self-selection. We predicted that participants would select into the selfish split default with the intention of switching to the fair split to send a stronger positive signal to observers.

\section{Method}

We anticipated that this task would be more difficult for participants to understand, and decided in advance to increase our target sample size to 250 participants per condition. A total of 506 undergraduates at UCSD's Rady School of Management $\left(M_{\text {age }}=20.84\right.$ years, one did not report age; $52 \%$ female, one reported "Other") participated for partial course credit. All participants played a modified Dictator Game as the dictator and were told that one randomly selected participant would have her choices played out for real money at the end of the experiment.

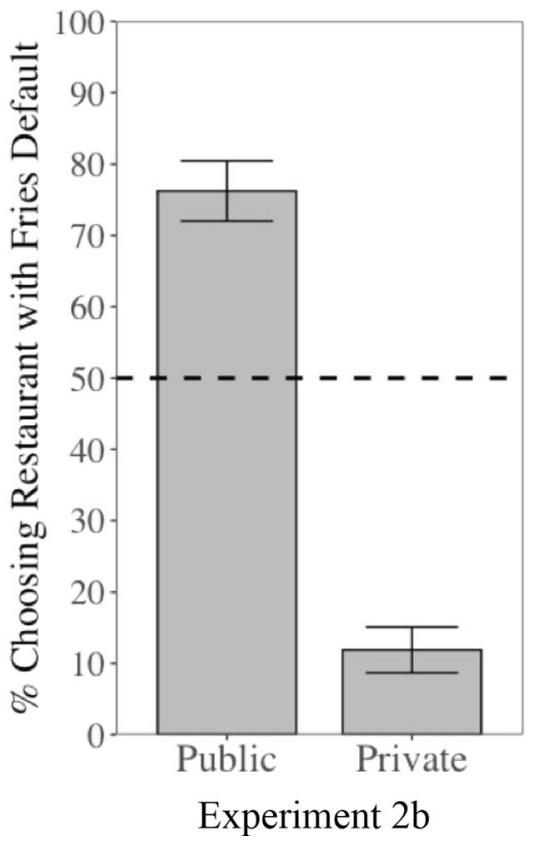

visibility of choice by either having dinner with a friend or alone. Error bars represent standard error of the proportion

In the modified Dictator Game, participants were endowed with $\$ 10$ and chose between a selfish split $(\$ 10, \$ 0)$ and a fair split $(\$ 5, \$ 5)$. In addition, participants chose between playing two versions of the game: In one version, the selfish split is the default, and in the other version, the fair split is the default. Participants watched four short clips that depict the four possible combinations of default and split, and selected one clip to represent their choices (see here for the four clips: https:/osf.io/ 7qpm5/).

Participants were randomly assigned to one of two conditions. In the "public" condition, participants were told that their choices in the Dictator Game would be shown to a separate group of third-party observers, and these observers would determine whether participants have the chance to play a subsequent Trust Game. ${ }^{2}$ Thus, through their choices in the Dictator Game, participants competed against each other to be selected by observers for the opportunity to earn additional money. The "private" condition serves as the control condition, and is identical to the "public" condition, except there were no observers and no opportunity to earn additional money in a subsequent Trust Game.

\footnotetext{
${ }^{2}$ In the Trust Game, Player 1 (the third-party observer) is endowed with $\$ 10$ and can send any of that amount to Player 2 (her chosen partner). Any amount that Player 1 sends to her partner is tripled. Player 2 can then return any of the amount she received back to Player 1 . At the end of the experiment, one randomly selected pair played the Trust Game for real money.
} 


\section{Results}

We first examined the participants' choice of split. While $45.1 \%$ (114/253) of participants in the private condition chose the fair split, $68.4 \%(173 / 253)$ of participants in the public condition did so, $\chi^{2}(1, N=506)=27.08, p<.001, \varphi=.23$. This suggests that participants believed that the fair split sends a more positive signal to observers than the selfish split. More importantly, we predicted that to amplify this positive signal, participants would pair this fair split with the selfish split default. Indeed, of those who chose the fair split, $67.1 \%$ $(116 / 173)$ of participants in the public condition chose the selfish split default, compared with only $52.6 \%(60 / 114)$ in the private condition, $\chi^{2}(1, N=287)=5.43, p=.02, \varphi=.14$ (see Fig. 3). Moreover, of those who chose the fair split, the percentage of participants who chose the selfish split default is significantly higher than chance (i.e., 50\%) in the public condition, $\chi^{2}(1, N=173)=19.45, p<.001,95 \%$ CI $[59.4 \%$, $73.9 \%$, but not in the private condition, $\chi^{2}(1, N=114)=$ $0.22, p=.64,95 \%$ CI $[43.1 \%, 62.0 \%]$.

We also explored whether those who chose the selfish split would be more likely to choose the selfish split default to avoid sending a stronger negative signal. On the contrary, of those who chose the selfish split, 70.0\% (56/80) of participants in the public condition chose the selfish split default, compared with $84.9 \%(118 / 139)$ in the private condition, $\chi^{2}(1, N=219)=6.02, p=.014, \varphi=.17$. We speculate that these participants may not have cared about their image, and

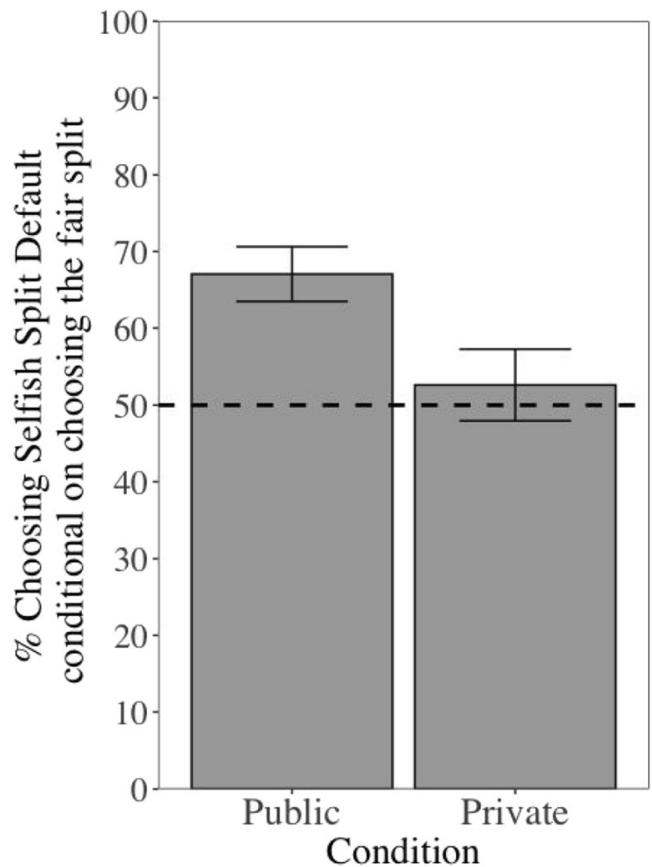

Fig. 3 Of those who chose the fair split, the percentage of participants in the private and public conditions who chose the selfish split default (rather than the fair split default) in Experiment 3. Error bars represent standard error of the proportion therefore did not have the goal to avoid sending a stronger negative signal.

To ensure that the fair split paired with the selfish split default in fact sends a stronger positive signal, we ran another study that placed participants in the role of third-party observers $(N=157)$. When presented with the same four clips that depicted the possible combinations of default and split, $60.5 \%(95 / 157)$ of participants selected the dictator who implemented the fair split under the selfish split default as their partner in the Trust Game, $\chi^{2}(1, N=157)=103.7, p<.001$, $95 \%$ CI $[52.4 \%, 68.1 \%]$ (against chance of $25 \%$ ). Together, these results provide further evidence that participants can anticipate how their choices will be perceived and can strategically select into default regimes with the intention of switching from the default to send a stronger signal.

\section{Experiment 4}

So far, we have focused on choices that convey positive signals, but choices can also convey negative signals (e.g., Young, Monin, \& Owens, 2009). When choices communicate negative signals, DMs should avoid sending stronger negative signals by choosing the default regime where they can simply stay with the default. In our final experiment, we further explored this hypothesis by manipulating both the signal and the visibility of choice. In a hypothetical grocery bag scenario, participants imagined shopping while using either their own reusable bags or store-provided plastic bags and either with a friend or alone. We predicted that participants would be more likely to choose a store where plastic bags were the default when shopping with a friend than when shopping alone, both to enhance a positive signal when using reusable bags (by switching from the store's default) and to attenuate a negative signal when using plastic bags (by staying with the store's default).

\section{Method}

Using a rule of thumb, we decided in advance to set our target sample size to be 150 participants per condition. A total of 603 Amazon Mechanical Turk workers participated in exchange for $\$ 0.20$, and were randomly assigned to one of four conditions. After excluding 35 participants who failed the attention check, we were left with a final sample of 568 participants $\left(M_{\mathrm{age}}=36.30\right.$ years; $48 \%$ female, one reported "Other"). Participants imagined that they were going grocery shopping and could choose one of two stores. The two stores were said to be comparable in every way, except customers are provided with plastic bags by default at one store and are asked to make an active choice between plastic and reusable bags at the other (no default). In the "positive signal" conditions, participants were told that they are using their own reusable bags, whereas 
in the "negative signal" conditions they were told that they are using store-provided plastic bags. In addition, participants in the "public" conditions are shopping with a friend who is environmentally conscious, and this friend would only know about the store where they shop. Participants in the "private" conditions are instead shopping alone.

\section{Results}

Figure 4 shows the percentage of participants who chose the store with the plastic bag default for each of the four conditions. The left bars correspond to staying with the default to avoid sending a stronger negative signal, and the right bars correspond to switching from the default to send a stronger positive signal. We performed a general linear regression with signal and visibility as the independent variables, and the participants' binary choice of store as the dependent variable $(1=$ plastic bag default, $0=$ no default). As predicted, participants were more likely to choose the plastic bag default store in the public condition than in the private condition, 52.7\% (146/ 277) versus $39.5 \%(115 / 291), \chi^{2}(1)=4.18, p=.041$. Furthermore, participants were more likely to choose the plastic bag default store in the negative signal condition than in the positive signal condition, $67.8 \%$ (192/283) versus $24.2 \%$ (69/ $285), \chi^{2}(1)=63.37, p<.001$. That is, they were more likely to choose the plastic bag default setting when it matched their use of plastic bags. More importantly, the signal-by-visibility interaction was not significant, $\chi^{2}(1)=0.39, p=.53$. In other words, we did not find evidence that the effect of visibility depends on the valence of the signal. These results suggest that those who care about sending a positive signal and those who care about avoiding a negative signal both prefer the same default setting, the former to enhance their signal by switching and the latter to attenuate their signal by staying.

\section{General discussion}

Unlike past studies that randomly assigned participants to different defaults, our paradigm allowed participants to choose their own default setting. Using healthy eating (Experiments $2 \mathrm{a}$ and $2 \mathrm{~b}$ ) and grocery bag (Experiment 4) scenarios as well as an incentivized economic game (Experiment 3), we found that participants were more likely to self-select into different default settings in the presence of observers. Specifically, they were more likely to choose default settings that require them to switch when sending a positive signal, and to choose default settings that require them to stay when sending a negative signal. Furthermore, the combination of default and choice that participants selected matches the one that observers actually perceive as indicating a stronger positive signal (Experiments 1 and 3). Taken together, these results suggest that DMs understand how their choices under different defaults are construed by others and can choose the optimal combination to enhance a positive signal or attenuate a negative signal.

Our results challenge the claims of default neglect by Zlatev et al. (2017). Rather than failing to exploit the influence that defaults have on others' choices, DMs appear to have a sophisticated understanding of what their choices signal under different defaults and can use this to their advantage in impression management. This provides convergent evidence that

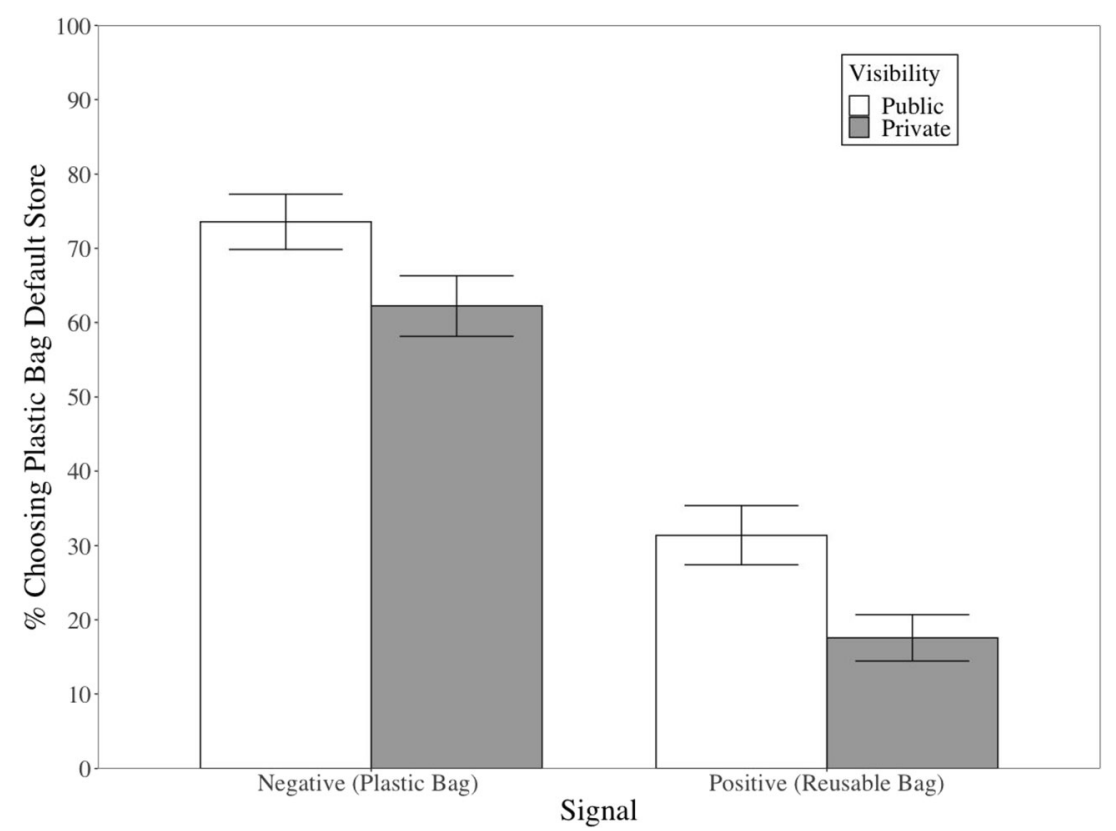

Fig. 4 The percentage of participants who chose the store with the plastic bag default as a function of the valence and visibility of choice in Experiment 4 . Error bars represent standard error of proportion 
default neglect may not be a general phenomenon, but rather is restricted to Zlatev et al.'s original materials (see also McKenzie et al., 2019).

Although choice architecture is conventionally discussed as a way to leverage cognitive biases for good, our findings support the framework of choice architecture as an implicit social interaction (Krijnen et al., 2017; McKenzie, Sher, Leong, \& Müller-Trede, 2018; Sher \& McKenzie, 2006). In this framework, choice architecture can influence DMs in two ways: (1) by changing the signal that their choice conveys and (2) through the inferences that they draw from the decision context. First, the same choice that requires switching rather than staying with the default is perceived by observers as more meaningful and impactful (Davidai et al., 2012). Our experiments complement this work by reversing the roles and allowing DMs to select the default setting. When given this opportunity, DMs were able to strategically exploit the asymmetric signal to influence others' perceptions. Second, previous studies have demonstrated that default effects occur in part because DMs perceive defaults as implicit recommendations (McKenzie, Liersch, \& Finkelstein, 2006). The choice of default can "leak" the default setter's personal attitudes and intentions, and DMs are sensitive to this cue. Thus, DMs appear to consider the social aspect of the choice context, both in terms of what their choices in that context may reveal to observers and the information that the context may implicitly convey.

Our findings have potentially important implications. Much evidence has shown that an effective way to promote a certain option is to set that option as the default. Our results caution that such defaults may sometimes backfire by driving away DMs who want to choose that option, but whose positive signal is diluted by staying with the default. These DMs will prefer default settings where they can switch from the default to send a stronger signal. But before generalizing our findings to real-world settings, a few limitations are in order. First, in our experiments, participants were explicitly given the goal to signal, and we do not know how often DMs have a strong motivation to signal outside the laboratory. Second, the DM's choice between different default settings could itself send a signal if observers were aware of the choice. In our experiments, participants were explicitly told that the observer would not know about their choice between different default settings, but only the one they end up choosing. The conditions under which these assumptions are met and whether this backfiring would occur in typical real-world default settings can be explored in future field studies.

Future research can also investigate the mechanisms for why switching from the default is perceived as a stronger signal than staying. Promising starting points are the theoretical explanations for why default effects occur: loss aversion, effort, and implicit recommendation (Jachimowicz, Duncan, Weber, \& Johnson, 2019). If observers believe that people tend to stick with defaults because doing so is less effortful, then switching could indicate a stronger preference because it is costly. Or if observers believe that people tend to stick with defaults because it is the recommended option, then switching could indicate that DMs have good reasons to reject this recommendation. Another interesting question that arises then is whether the factor that drives observer perception matches the one that DMs consider when choosing.

In sum, people care about what their choices communicate to others. Under different default settings, choices that lead to identical outcomes may nevertheless convey different social meanings. When people can select their choice environments, they do so strategically to exploit the asymmetric signals that observers perceive from choices under different defaults.

\section{References}

Benartzi, S., Beshears, J., Milkman, K. L., Sunstein, C. R., Thaler, R. H., Shankar, M., ... Galing, S. (2017). Should governments invest more in nudging? Psychological Science, 28, 1041-1055. doi:https://doi. org/10.1177/0956797617702501

Davidai, S., Gilovich, T., \& Ross, L. D. (2012). The meaning of default options for potential organ donors. Proceedings of the National Academy of Sciences of the United States of America, 109, 1520115205. doi:https://doi.org/10.1073/pnas.1211695109

Jachimowicz, J. M., Duncan, S., Weber, E. U., \& Johnson, E. J. (2019). When and why defaults influence decisions: A meta-analysis of default effects. Behavioural Public Policy, 1-28. doi:https://doi. org/10.1017/bpp.2018.43

Johnson, E. J., \& Goldstein, D. (2003). Do defaults save lives? Science, 302, 1338-1339. doi:https://doi.org/10.1126/science.1091721

Jung, M. H., Sun, C., \& Nelson, L. D. (2018). People can recognize, learn, and apply default effects in social influence. Proceedings of the National Academy of Sciences of the United States of America, 115(35), E8105-E8106. doi:https://doi.org/10.1073/pnas. 1810986115

Krijnen, J. M. T., Tannenbaum, D., \& Fox, C. R. (2017). Choice architecture 2.0: Behavioral policy as an implicit social interaction. Behavioral Science \& Policy, 3(2), 1-18. doi:https://doi.org/10. 1353/bsp.2017.0010

Lin, Y., Osman, M., Harris, A. J., \& Read, D. (2018). Underlying wishes and nudged choices. Journal of Experimental Psychology: Applied, 24(4), 459-475. doi:https://doi.org/10.1037/xap0000183

McKenzie, C. R. M., Liersch, M. K., \& Finkelstein, S. R. (2006). Recommendations implicit in policy defaults. Psychological Science, 17, 414-420. doi:https://doi.org/10.1111/j.1467-9280. 2006.01721.x

McKenzie, C. R. M., Leong, L. M., \& Sher, S. (2019). Default sensitivity in attempts at social influence. Manuscript submitted for publication.

McKenzie, C. R. M., Sher, S., Leong L. M., \& Müller-Trede, J. (2018). Constructed preferences, rationality, and choice architecture. Review of Behavioral Economics, 5, 337-360. doi:https://doi.org/10.1561/ 105.00000091

Pleasant, A., \& Barclay, P. (2018). Why hate the good guy? Antisocial punishment of high cooperators is greater when people compete to be chosen. Psychological Science, 29(6), 868-876. doi:https://doi. org/10.1177/0956797617752642 
Sher, S., \& McKenzie, C. R. M. (2006). Information leakage from logically equivalent frames. Cognition, 101, 467-494. doi:https://doi. org/10.1016/j.cognition.2005.11.001

Thaler, R. H., \& Sunstein, C. R. (2008). Nudge: Improving decisions about health, wealth and happiness. London, England: Penguin Books.

Young, S. D., Monin, B., \& Owens, D. (2009). Opt-out testing for stigmatized diseases: A social psychological approach to understanding the potential effect of recommendations for routine HIV testing.
Health Psychology, 28(6), 675-681. doi:https://doi.org/10.1037/ a0016395

Zlatev, J. J., Daniels, D. P., Kim, H., \& Neale, M. A. (2017). Default neglect in attempts at social influence. Proceedings of the National Academy of Sciences of the United States of America, 114(52), 13643-13648. doi:https://doi.org/10.1073/pnas.1712757114

Publisher's note Springer Nature remains neutral with regard to jurisdictional claims in published maps and institutional affiliations. 